\title{
Effects of platelet-rich plasma on in vitro proliferation and migration of fibroblasts from human chronic refractory wound granulation tissue
}

\author{
Tie-ning Zhang ${ }^{1,2}$, Quan $\mathrm{Li}^{* 2}, \mathrm{Te} \mathrm{Ba}^{2}$, Tian-xi Shao ${ }^{1,2}$, Fang $\mathrm{Li}^{2}$, Ling-feng Wang ${ }^{2}$ \\ ${ }^{1}$ Baotou Medical College of Inner Mongolia University of Science and Technology, Baotou, Inner Mongolia, China \\ ${ }^{2}$ Department of Burns, Baogang Hospital, Baotou, Inner Mongolia, China
}

Received: March 30, 2021

DOI: $10.5430 /$ dcc.v8n1p24
Accepted: July 25, 2021

URL: https://doi.org/10.5430/dcc.v8n1p24
Online Published: August 18, 2021

\begin{abstract}
Objective: To observe the effects of platelet-rich plasma (PRP) on in vitro proliferation and migration of fibroblasts from human chronic refractory wound granulation tissue.

Methods: Fibroblasts were separated from human chronic refractory wound granulation tissue and then were identified. The obtained fibroblasts were divided into fetal bovine serum (FBS) group, hydrogel group and PRP group, and the three groups were cultured with culture mediums containing FBS, hydrogel and PRP respectively, in order to observe the growth of fibroblasts. The wound scratch assay was used to observe the migration of fibroblasts.

Results: PRP group had more fibroblasts than FBS group and hydrogel group since Day 5 of culture, and exhibited greater fibroblast scratch migration area than FBS group on $48 \mathrm{~h}$ and $72 \mathrm{~h}$ of wound scratch assay (all $p<.05$ ).

Conclusions: Compared with FBS, human fibroblasts cultured by PRP can more effectively promote the proliferation and migration of fibroblasts.
\end{abstract}

Key Words: Fibroblast, Chronic refractory wound, Granulation tissue, Platelet-rich plasma, Cell proliferation, Cell migration, In vitro

\section{INTRODUCTION}

Chronic refractory wound refers to the wound which is caused by various factors and not healed even with no obvious healing tendency after more than one-month treatment. ${ }^{[1]}$ With the development of domestic social economy, the aging of the population and the changing of citizen lifestyle, chronic disease spectrum has changed and the incidence of chronic refractory wound has been increased accordingly. Currently, the major treatment options for chronic refractory wound consist of surgical treatment, dressing change, nega- tive pressure drainage and so on. Nevertheless, these options have a long-time treatment course with high costs and poor effects. ${ }^{[2,3]}$ Platelet-rich plasma (PRP), as a research focus of tissue engineering, provides a new idea for the treatment of chronic refractory wound. This research is designed to isolate fibroblasts from human chronic refractory wound granulation tissue to observe and compare the biological behaviors of fibroblasts cultured in different culture mediums, providing a theoretical foundation for the treatment of chronic refractory wound by use of PRP.

*Correspondence: Quan Li; Email: Btyxyztn@ 163.com; Address: Department of Burns, Baogang Hospital, Baotou, Inner Mongolia 014010, China. 


\section{MAterials AND MethodS}

\subsection{Source of samples, main instruments and reagents}

\subsubsection{Source of samples}

The post-operative abandoned granulation tissues from patients who were given chronic wound debridement in Chronic Wound Diagnosis and Treatment Management Center of Baogang Hospital, with $50 \mathrm{ml}$ of venous blood taken from each of the mentioned-above patients. Inclusion criteria: (1) patients aged from 18 to 65 ; (2) patients with the wound not healed more than one month and the wound area not reduced in the past 2 weeks; (3) patients with the wound in the non-acute infective stage (such as obvious redness, swelling, hotness, many purulent secretions or obvious odors); (4) patients with full thickness wound caused by various factors, such as III degree burn, severe trauma and so on. All patients were informed of the sampling issues and required to sign informed consent forms.

\subsubsection{Main reagents and instruments}

The medical centrifuge and PRP preparation kits were purchased from WEGO Medical Instrument (Shandong) Co., Ltd. (Filing No.: 20150044); sodium alginate, type I collagenase, trypsin-EDTA, anhydrous calcium chloride and human type I collagenase ELISA kits were bought from Sigma (Kit Batch No.: W201502, SCR103, 59418C, C1016 and CC050); Dulbecco's modified Eagle medium (DMEM) and fetal bovine serum (FBS) were merchand from ised Gibco (Kit Batch No.: A4192101, 12664025).

\subsubsection{The preparation of PRP and hydrogel}

PRP preparation kits were used to prepare PRP. Whole blood specimens were taken from each patient, centrifuged at the rotate speed of $4,000 \mathrm{r} / \mathrm{min}$ at the room temperature, with the bottom layer of erythrocytes abandoned. The remained sample was then centrifuged (at the rotate speed of 4,000 $\mathrm{r} / \mathrm{min}$, room temperature). The brownish yellow layer in the middle was PRP. See Figure 1 for details. See Ref. ${ }^{[4]}$ for the preparation of hydrogel.
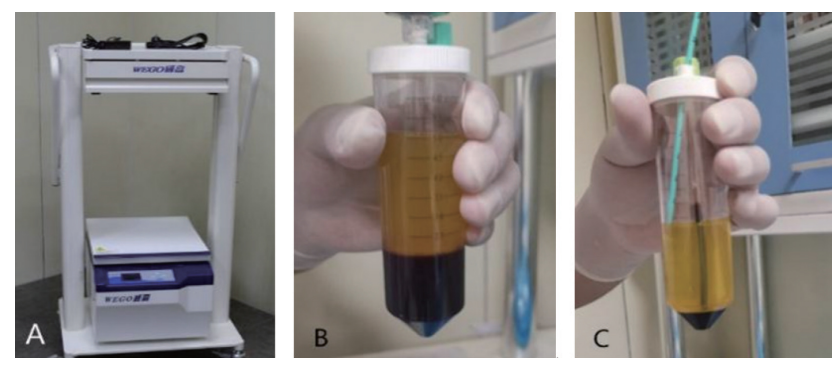

Figure 1. The preparation of PRP

A. Medical Centrifuge; B. First Centrifugation Results; C. Second Centrifugation Results

\subsection{Experimental methods}

\subsubsection{Isolation and identification of fibroblasts}

Chronic refractory wound granulation tissues were collected from the patients in these three groups. Under the aseptic condition, phosphate buffered saline (PBS) containing double antibodies (penicillin $100 \mathrm{U} / \mathrm{ml}$ and streptomycin 100 $\mathrm{U} / \mathrm{ml}$ ) (Sigma) was used to rinse out thoroughly to remove pollutants, with the tissue cut into a square of $2 \mathrm{~mm} \times 2 \mathrm{~mm}$. Subsequently, the tissue was rinsed out three times by use of double-antibody PBS, cut into pieces and then transferred to the centrifuge tube containing $0.1 \%$ type I collagenase (1:2). The constant-temperature shaking incubator (set at $37^{\circ} \mathrm{C}$ ) was used to oscillate and digest these tissues for $2 \mathrm{~h}$. After the cells could be observed under the microscope, the equivalent volume of DMEM solution (containing FBS) was added in order to terminate the digestion. The cell filtrate was collected and centrifuged at the rotate speed of 1,500 $\mathrm{r} / \mathrm{min}$ for $10 \mathrm{~min}$, with the supernatant abandoned. Subsequently, it was resuspended in DMEM solution containing FBS, in order to acquire the target cells. These cells were seeded in T25 culture bottle at a density of $1 \times 10^{5}$ cells $/ \mathrm{ml}$. If the proportion of primary cell fusion approximated $100 \%$, the culture medium was then discarded. Trypsin-EDTA was added for the use of digestion, and the digestion solution was abandoned after the digestion was finished. EDTA was added to slightly dissociate the adherent cells into single cells, which were inoculated in 1:2 for serial passage. After $20 \mathrm{~h}$, the cells proliferated adherently and covered the bottom of the culture bottle. Cells in the third passage were chosen and placed under the microscope to observe the general morphology. The immunohistochemical method was used to detect vimentin (vimentin antibodies were purchased from Abcam of Britain, Batch No.: ab92547) to identify fibroblasts.

\subsubsection{Determination of fibroblast growth curves}

The third passage of fibroblasts were collected and divided into FBS group, hydrogel group and PRP group. The three groups of fibroblasts were cultured continuously in FBS, hydrogel and DMEM-PRP for 8 days, and the concentration of fibroblasts in each group was $10^{3}$ cells/well. A small amount of culture was taken from the culture medium in each group, stained with crystal violet and counted by use of blood counting chamber. The measurement was made three times in each group every day, and the results were averaged and used to plot cell growth curves. The experiment was made in quintuplicate.

\subsubsection{Wound scratch assay}

The third passage of fibroblasts were collected and divided into FBS group, hydrogel group and PRP group. They were inoculated in 12-well cell culture plates respectively with a seeding density of $5 \times 10^{5}$ cells/well. When the cells prolif- 
erated adherently and covered the whole well, according to Ref., ${ }^{[5]} 200 \mu$ l pipette was used to scratch the bottom of the culture plate, in order to observe the cell fusion on the time points of $24 \mathrm{~h}, 48 \mathrm{~h}$ and $72 \mathrm{~h}$ of the wound scratch assay. The experiment was made in quintuplicate. The cell migration experiment was not made in hydrogel group.

\subsection{Statistical analysis}

SPSS 17.0 software was applied to statistical analysis. The measurement data were represented by $(\bar{x} \pm \mathrm{s})$. The comparison between two groups was made by use of $t$-test, and the comparison among groups was made by one way ANOVA. The difference $p<.05$ was of statistical significance.

\section{RESULTS}

\subsection{The morphology and identification of fibroblasts}

When the isolated fibroblasts just proliferated adherently, the cellular morphology showed elongated or irregular shape. Later, the passage cells changed their morphology into homogeneous shape, with a regular arrangement. When the cell concentration was low, these cells arranged loose; when the concentration was high, the cells arranged in parallel or spiral. The cell body was long and narrow, lucent, with abundant cytoplasm; the cell nucleus was clear, oval, mediate, with dikaryon occasionally observed. The immunohistochemical staining results showed that the cell cytoplasm was brownish yellow and the cell nucleus was mauve. It was indicated that the harvested cells were fibroblasts. See Figure 2 for details.

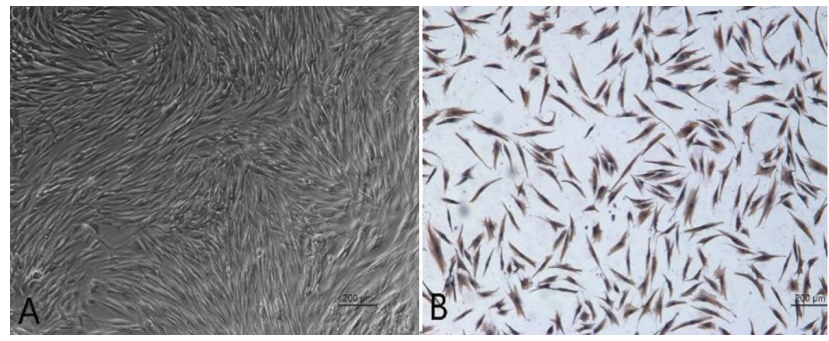

Figure 2. The morphology and immunohistochemical experiment results of fibroblasts from human chronic refractory wound granulation tissue

A. Passage Fibroblast Morphology $(\times 50)$; B. Fibroblast

Immunohistochemical Staining Results $(\times 50)$

\subsection{The proliferation of fibroblasts in the three groups}

All fibroblasts in the three groups grew well. On Day 2, the cells grew faster; on Day 3 to Day 5, the cells proliferated most actively. On Day 5, the growth of the cells in FBS group reached their highest point, and the cells grew slower on Day 6 to Day 8. In hydrogel group, the growth of the cells reached their peak on Day 6, and the cells grew slower on Day 7 to Day 8. The cells in PRP group proliferated continuously. See Figure 3 for details. On Day 5 to Day 8, the amount of the cells in PRP group was larger than that in FBS group and hydrogel group respectively. On Day 6 and Day 8 , the amount of the cells in hydrogel group was larger than that in FBS group (all $p<.05$ ). On the other time points, there were no statistically significant differences in the cell counts in the three groups (all $p>.05$ ). See Table 1 for details.

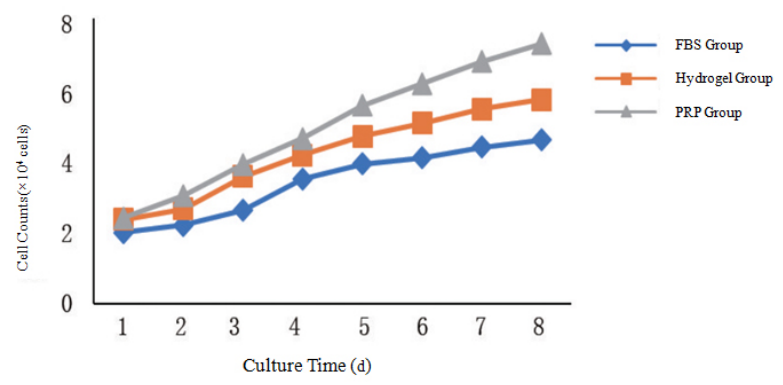

Figure 3. Growth curves of fibroblasts in different culture mediums

\subsection{Comparison in the migration area in the wound scratch assay between two groups of fibroblasts}

On the time point of $24 \mathrm{~h}$ of the wound scratch assay, there was no statistically significant difference in the comparison of cell migration area between PRP group and FBS group ( $p$ $>.05$ ); on the time points of $48 \mathrm{~h}$ and $72 \mathrm{~h}$, the cell migration area of PRP group was greater than that of FBS group (all $p$ $<.05)$. See Table 2 and Figure 4 for details.

\section{Discussion}

Fibroblasts are major repair cells for wound healing. If their physiological function is inhibited, ${ }^{[2,3]}$ it will affect the normal wound healing, even induce the formation of chronic refractory wound. How to recover the normal physiological function of fibroblasts becomes a new idea for the treatment of chronic refractory wound. On account of easy-to-get, normal skin tissues or cicatricial tissues were used as specimens in past researches on fibroblasts. Nevertheless, they cannot reflect the characteristics of skin tissues from chronic wound. In this research, chronic refractory wound granulation tissue was directly collected to isolate fibroblasts successfully for the follow-up experiment.

La et al. ${ }^{[6]}$ have found that FBS can promote the proliferation of human skin fibroblasts in vitro. There are also some other researches showing that PRP can promote the growth and migration of fibroblasts. ${ }^{[-9]}$ PRP is a type of platelet concentrate extracted from the blood. Some researches have confirmed that ${ }^{[3,7-11]}$ PRP contains fibroblast growth factor, platelet-derived growth factor, epidermal growth factor, 
vascular endothelial growth factor, insulin-like growth factor, transforming growth factor and other growth factors. All these growth factors in PRP can promote wound healing. ${ }^{[12-14]}$ Currently, PRP has been widely applied in Department of Plastic Surgery, Department of Orthopaedics and other departments. ${ }^{[10,15]}$ To explore the therapeutical effect of PRP on chronic refractory wound, in this research, fibroblasts were cultured in different culture mediums containing FBS, hydrogel and PRP respectively, in order to observe the cell migration. The research results showed that, since Day 5, the number of fibroblasts in PRP group was larger than that in FBS group and hydrogel group respectively. On the time points of $48 \mathrm{~h}$ and $72 \mathrm{~h}$, the cell migration area of PRP group was greater than that of FBS group (all $p<$
$.05)$. It is indicated that, in comparison with FBS, PRP can better promote the proliferation and migration of fibroblasts from human chronic refractory wound granulation tissue, which is similar to the research results from Cho et al. ${ }^{[7-9]}$ It is because PRP in the plasma can provide part of nutrient substances, electrolytes, hormones and growth factors for platelets and fibroblasts, and then regulate the physiological activities of platelets and cells. Simultaneously, platelets can continuously and slowly release a variety of growth factors and promote the cell proliferation. It is indicated that PRP can stimulate wound healing by influencing the growth and migration of fibroblasts from the wound. It provides a new idea for the treatment of chronic refractory wound by use of PRP.

Table 1. Comparison in the proliferation of fibroblasts among the three groups $\left(\bar{x} \pm \mathrm{s}, \times 10^{4}\right.$ cells $)$

\begin{tabular}{llllllllll}
\hline Group & n & Day 1 & Day 2 & Day 3 & Day 4 & Day 5 & Day 6 & Day 7 & Day 8 \\
\hline FBS Group & 5 & $2.0 \pm 0.8$ & $2.2 \pm 0.7^{\&}$ & $2.6 \pm 0.6$ & $3.5 \pm 0.6$ & $3.9 \pm 0.6^{\&}$ & $4.1 \pm 0.7^{\&}$ & $4.4 \pm 0.6^{\&}$ & $4.6 \pm 0.7^{\&}$ \\
Hydrogel Group & 5 & $2.3 \pm 0.4$ & $2.7 \pm 0.7$ & $3.6 \pm 0.8$ & $4.2 \pm 0.7$ & $4.7 \pm 0.7^{\&}$ & $5.1 \pm 0.8^{* \&}$ & $5.5 \pm 1.0^{\&}$ & $5.7 \pm 0.9^{* \&}$ \\
PRP Group & 5 & $2.4 \pm 0.5$ & $3.0 \pm 0.7$ & $3.9 \pm 0.9$ & $4.6 \pm 0.7$ & $5.6 \pm 0.5$ & $6.2 \pm 0.6$ & $6.8 \pm 0.8$ & $7.3 \pm 0.5$ \\
F Value & & 0.619 & 1.667 & 3.840 & 3.470 & 9.864 & 11.107 & 10.825 & 17.839 \\
$p$ Value & & .555 & .230 & .051 & .065 & .003 & .002 & .002 & $<.001$ \\
\hline
\end{tabular}

Note. in comparison with FBS group, ${ }^{*} p<.05$; in comparison with PRP group, ${ }^{8} p<.05$

Table 2. The comparison in the cell migration area in the wound scratch assay between two groups of fibroblasts $(\bar{x} \pm \mathrm{s}, \%)$

\begin{tabular}{lllll}
\hline Group & $\mathbf{n}$ & $\mathbf{2 4} \mathbf{h}$ & $\mathbf{4 8 ~ h}$ & $\mathbf{7 2} \mathbf{~ h}$ \\
\hline FBS Group & 5 & $11.4 \pm 5.7$ & $61.2 \pm 8.2$ & $72.6 \pm 8.4$ \\
PRP Group & 5 & $10.2 \pm 4.5$ & $79.4 \pm 6.8$ & $94.6 \pm 4.3$ \\
t Value & & 0.369 & 3.820 & 5.213 \\
$p$ Value & & .721 & .005 & .001 \\
\hline
\end{tabular}

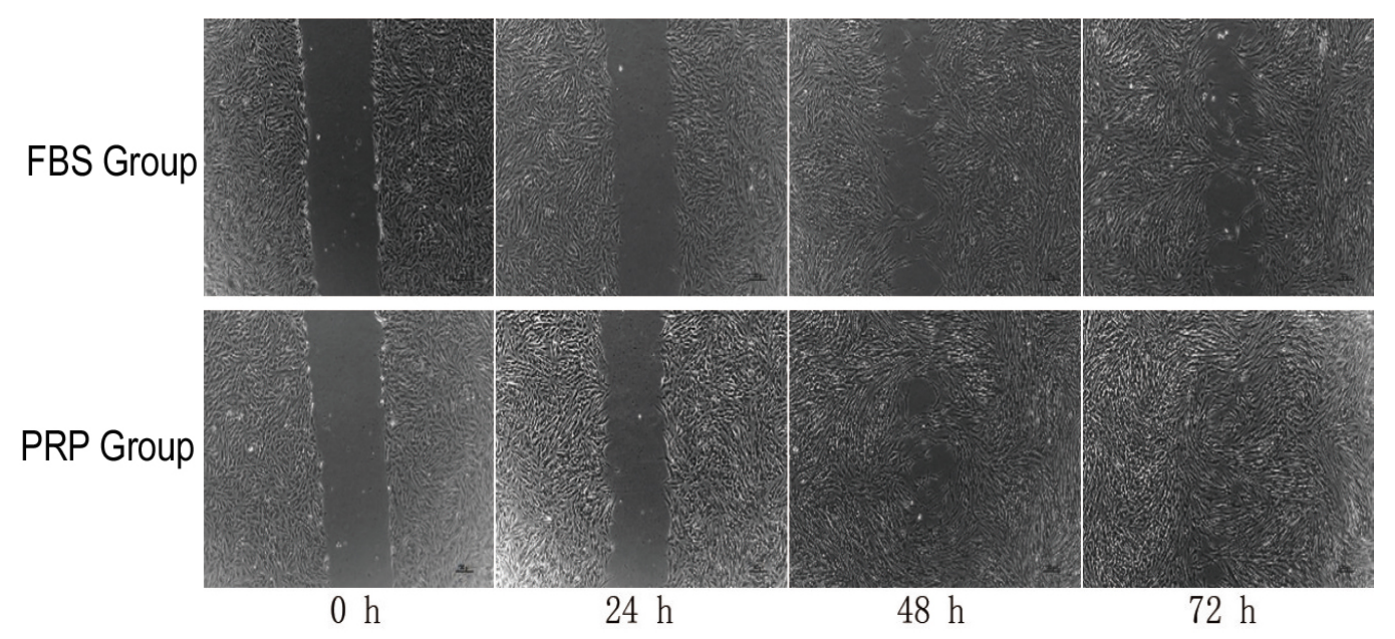

Figure 4. Fibroblast wound scratch assay $(\times 50)$ 
In this research, PRP preparation kits were applied to the twostep centrifugation in order to prepare PRP. During the process of preparation, it is no need to replace centrifuge tubes. On that account, the activation and pollution of platelets was reduced correspondingly, with part of plasma ingredients reserved simultaneously. Meanwhile, in comparison with the conventional tissue engineering materials, autologous blood preparation can be adopted to prepare PRP, ${ }^{[11]}$ with easy operation. It reduces the potential biological risks brought by allogenic or xenogeneic materials, and relieve patients' burden. It provides great convenience for the clinical application of PRP. However, in the clinical application of PRP, it is needed to take the following issues into consideration: (1) the amount of one-time prepared PRP is not enough for the whole course of treatment, then how to effectively prolong the in-vitro reservation time of PRP, avoid repeated blood sampling and relieve patients' pain; (2) Whether and how PRP can be applied in combination with antibiotics and their effects; (3) whether the application of PRP can bring the risk for hyperplasia and cicatrization during wound healing. All these issues need to be further studied.

In conclusion, in comparison with FBS, human fibroblasts cultured by PRP can more effectively promote the proliferation and migration of fibroblasts. It provides a new theoretical foundation for the treatment of chronic refractory wound by use of PRP.

\section{CONFlicts OF INTEREST Disclosure}

The authors declare they have no conflicts of interest.

\section{REFERENCES}

[1] Liao X, Guo G. Classification and clinical evaluation of chronic refractory wounds. Chinese Journal of Injury Repair and Wound Healing (Electronic Edition). 2017; 12(04): 303-305.

[2] Zhang C, Wang M, Chai Y. Research progress on the effects of platelet-rich plasma on chronic refractory wound. International Journal of Orthopaedics. 2019; 40(2): 100-103.

[3] Wang J, Hui Q, Guo B, et al. Application of platelet-rich plasma to the treatment of chronic wound. Chinese Journal of Aesthetic and Plastic Surgery. 2017; 28(1): 63-65.

[4] Zhao D, Tie C, Wang X, et al. Effects of adding strontium ions into photocrosslinked alginate hydrogels on its mechanical and biological properties. Chinese Journal of Tissue Engineering Research. 2019; 23(18): 2880-2887.

[5] Li Q, Cui F, Ba T, et al. Preliminary study of promotion effect of adipose derived stem cell-exosomes on fibroblast proliferation derived from granulation tissues. Chinese Journal of Injury Repair and Wound Healing (Electronic Edition). 2019; 14(2): 91-96.

[6] La W, Lin X, Jiang W, et al. The effects of different culture medium on the proliferation, aging and collagen secretion of fibroblasts from skin. Chinese Journal of Aesthetic Medicine. 2017; 26(2): 68-72.

[7] Cho EB, Park GS, Park SS, et al. Effect of platelet-rich plasma on proliferation and migration in human dermal fibroblasts. J Cosmet Dermatol. 2019; 18(4): 1105-1112. https://doi.org/10.1111/ jocd. 12780
[8] Tian Y, Zhang Q, Wang H, et al. Promotion of growth and migration of fibroblasts through platelet-rich plasma. Chinese Journal of Aesthetic and Plastic Surgery. 2019; 30(8): 487-490.

[9] Jia C, Chen L, Yang Q, et al. Effect of platelet-rich plasma on the proliferation of photoaging skin fibroblasts. Chinese Journal of Aesthetic and Plastic Surgery. 2016; 27(4): 234-237.

[10] Law JX, Chowdhury SR, Saim AB, et al. Platelet-rich plasma with keratinocytes and fibroblasts enhance healing of full-thickness wounds. J Tissue Viability. 2017; 26(3): 208-215. https ://doi .or $\mathrm{g} / 10.1016 / \mathrm{j} \cdot \mathrm{jtv} \cdot 2017.05 .003$

[11] Marques LF, Stessuk T, Cherici Camargo IC, et al. Platelet-rich plasma (PRP): methodological aspects and clinical applications. Platelets. 2015; 26(2): 101-113. https ://doi.org/10.3109/09 537104.2014 .881991

[12] Chen J, Xie B, Huang Y, et al. Effect of the Platelet Rich Plasma Combined with Debridement in the Treatment of Refractory Wounds. Chinese Journal of Aesthetic Medicine. 2018; 27(4): 11-14.

[13] Zhao Y, Zhu Z, Li A, et al. Clinical study on platelet-rich plasma in repairing chronic wound. Journal of Clinical Surgery. 2016; 24(3): 175-178.

[14] Giusti I, Di Francesco M, D’Ascenzo S, et al. Leukocyte depletion does not affect the in vitro healing ability of platelet rich plasma. Exp Ther Med. 2018; 15(4): 4029-4038. https ://doi.org/10.3892/ etm. 2018. 5887

[15] Yu J, Feng Y, Wu Y. Effect of delivery autologous platelet rich plasma into skin with microneedle for facial rejuvenation. Chinese Journal of Aesthetic Medicine. 2015; 24(17): 33-37. 